\title{
Stimulus History Alters Behavioral Responses of Neuronal Growth Cones
}

\author{
Thomas J. Diefenbach, Peter B. Guthrie, and Stanley B. Kater \\ Department of Neurobiology and Anatomy, University of Utah School of Medicine, Salt Lake City, Utah 84132
}

Generally, it is assumed that growth cones respond to a specific guidance cue with a single, specific, and stereotyped behavior. However, there is evidence to suggest that previous exposure to a given cue might alter subsequent responses to that cue (Snow and Letourneau, 1992; Shirasaki et al., 1998). We therefore tested the hypothesis that growth cone responses to stimuli are dependent on the history of previous stimulation. Growth cones of chick dorsal root ganglion neurons were exposed to well characterized stimuli: (1) contact with a laminin-coated bead, which causes growth cone turning, or (2) electrical stimulation, which causes growth cone collapse. Although the expected behavioral responses were observed after the initial stimulation, strikingly different responses to a subsequent stimulation were observed. Growth cones that had recovered from electrical stimulation-induced collapse rapidly developed insensitivity to a second identical electrical stimulation. Growth cones that previously turned in response to contact with a laminin-coated bead responded to a second bead with a "stall" or cessation in outgrowth. This stimulus history dependence of growth cone behavior could be generalized across dissimilar stimuli: after contact with a laminin-coated bead, growth cones failed to collapse in response to electrical stimulation. The calcium/calmodulin-dependent protein kinase II (CaMKII) was implicated in this history dependence by pharmacological experiments. Together, these results demonstrate that growth cones can alter their behavioral response rapidly to a given stimulus in a manner dependent on previous history and that knowledge of past events in growth cone navigation may be required to predict future growth cone behavior.

Key words: growth cone; laminin; collapse; dorsal root ganglion; guidance; CaMKII
During early development growth cones sequentially encounter multiple guidance cues during pathfinding (Bentley and Caudy, 1983; Caudy and Bentley, 1986; Singer et al., 1995; Davenport et al., 1996; Karlstrom et al., 1996; Melancon et al., 1997; Isbister et al., 1999). Such interactions are believed to lead to predictable, stereotyped behaviors defined primarily by the nature of the specific guidance cue. New evidence is emerging, however, that additional factors can modify the response to a given stimulus. For example, it is known that previous exposure to low concentrations of an inhibitory substrate can enable subsequent outgrowth onto higher, normally inhibitory, concentrations of the same substrate (Snow and Letourneau, 1992). Growth cones that initially have responded to a chemoattractant fail to respond to a subsequent exposure to that same attractant in situ (Shirasaki et al., 1998). One interpretation of such studies is that a previous encounter with a guidance cue can affect the future response of a growth cone to a subsequently encountered cue. This interpretation would require revision of the model of fixed, stereotypic growth cone responses to include a consideration of the previous stimulus history of the growth cone.

Several important issues arise from experiments on the effect

\footnotetext{
Received March 22, 1999; revised Oct. 29, 1999; accepted Dec. 1, 1999.

This research was supported through National Institutes of Health Grant NS24683 and a fellowship to T.J.D. from the Alberta Heritage Foundation for Medical Research (Alberta, Canada). We thank Dr. Maureen Condic for expert and helpful editorial comments and discussions. We also thank Mrs. Kathy Charters for assistance with culture preparation.

Correspondence should be addressed to Dr. Stanley B. Kater, Department of Neurobiology and Anatomy, University of Utah School of Medicine, 50 North Medical Drive, Salt Lake City, UT 84132. E-mail: Stanley.Kater@hsc.utah.edu.

Dr. Diefenbach's present address: Department of Physiology, Tufts University School of Medicine, 136 Harrison Avenue, Boston, MA 02111.

Copyright (C) 2000 Society for Neuroscience $\quad 0270-6474 / 00 / 201484-11 \$ 15.00 / 0$
}

of previous stimulus history on growth cone behavior. For example, can past exposure to a given stimulus alter growth cone responses to other, dissimilar stimuli? Furthermore, are the temporal details of stimulus presentation important? That is, is the interstimulus interval a potential parameter in interpreting cues a growth cone encounters during pathfinding? These questions are addressed in the present study, which examines the reactions of individual growth cones to discrete, sequentially presented stimuli.

An in vitro system of isolated chick dorsal root ganglion neurons was used to examine this potentially new view of growth cone behavior. With this system the growth cones could be presented with stimuli with a high degree of spatial and temporal precision. The two distinct kinds of stimuli used, presentation of laminin-coated beads and electrical stimulation, were chosen for their ability to elicit distinct growth cone behaviors reliably (Cohan and Kater, 1986; Cohan, 1990; Fields et al., 1990; Kuhn et al., 1995, 1998). We report here that individual growth cones indeed could alter their behavioral responses rapidly and consistently to stimuli in a history-dependent manner. Furthermore, such changes were observed after both similar and dissimilar stimuli and were highly dependent on the interval between stimuli.

\section{MATERIALS AND METHODS}

Cell culture. Primary cultures from lumbosacral dorsal root ganglia (DRG) of embryonic day (E) E9-E11 chick were prepared as described previously (Kuhn et al., 1995). Cells were plated on substrates of either fibronectin or laminin-1 (2 $\mu \mathrm{g} / \mathrm{cm}^{2}$; Collaborative Biomedical Research, Bedford, MA) on glass coverslips previously coated with poly-L-lysine (Kuhn et al., 1998). The culture medium consisted of MEM (Life Technologies, Gaithersburg, MD) with $10 \%$ fetal bovine serum (HyClone Laboratories, Hyrum, UT), $2 \mu \mathrm{g} / \mathrm{ml} \mathrm{2.5S}$ nerve growth factor (NGF; Collaborative Biomedical Research, Bedford, MA), and $\mathrm{N}_{3}$ sup- 
plement (Stoeckli et al., 1991). KN-93 and KT5720 were obtained from Calbiochem (La Jolla, CA). Tetrodotoxin (TTX) was obtained from Sigma (St. Louis, MO) and $\mathrm{NiCl}_{2}$ from Mallinckrodt (St. Louis, MO).

Electrical stimulation of growth cones. DRG neurons were plated in a modified Campenot chamber (Campenot, 1977) for electrical stimulation experiments. The chamber consisted of a halved nylon spacer adhered to the bottom of a glass-bottomed $35 \mathrm{~mm}$ Petri dish (Falcon, Oxnard, CA) with silicone vacuum grease (Dow Corning, Midland, MI). Before plating, laminin or fibronectin was added to both the inside and outside of the chamber. Growth cones could extend out of the chamber only under a \#0 glass coverslip partition ( $\sim 100 \mu \mathrm{m}$ thick) adhered to the open end of the chamber with silicone vacuum grease. A $20-40 \mu \mathrm{m}$ gap under the partition permitted the unimpeded extension of growth cones to the outside of the chamber. Platinum wires were placed inside and outside the chamber for stimulation. The resistance of the gap was $16-18 \mathrm{k} \Omega$.

Neurites passing under the glass partition were stimulated with $10 \mathrm{~Hz}$ trains of biphasic stimuli $(10 \mathrm{~V}, 200 \mu \mathrm{sec}$ duration), using an SD9 stimulator (Grass, Quincy, MA). Stimulation for $10 \mathrm{~min}$ was shown to be optimal for these experiments ( $82 \%$ collapse; 90 of 110). Shorter duration (5 min, $28 \%$ collapse; 8 of 29 ) or lower amplitude stimulation (6 V, $55 \%$ collapse; 6 of 11) resulted in reduced frequency of collapse. Longer duration stimulation $(15 \mathrm{~min})$ resulted in a slightly higher rate of collapse (92\% collapse; 11 of 12) but with significantly fewer growth cones recovering ( $27 \%$ recovery; 3 of 11 ) as compared with the 10 min stimulation (96\% recovery; 80 of 83 ). All observations were restricted to growth cones on neurites that passed under the coverslip partition.

Bead stimulation of growth cones. Laminin-coated beads were prepared from polystyrene beads (5 $\mu \mathrm{m}$ diameter) and laminin following the method of Kuhn and colleagues (1995). Beads were positioned ahead of growth cones by using a custom optical trapping system (Block, 1992) at $\sim 45^{\circ}$ angles with respect to the expected outgrowth direction of the growth cone. The positive response criterion after bead contact was a reorientation toward the bead of $>15^{\circ}$ (Kuhn et al., 1995). The specificity of interaction with laminin-coated beads had been tested previously by using beads coated with bovine serum albumin (Fraction V, Sigma), beads coated with laminin in reverse orientation, and bead preincubated with an anti-laminin antibody (Kuhn et al., 1995).

Monitoring growth cone behavior. Cultures were observed at $37^{\circ} \mathrm{C}$ in MEM with $5 \% \mathrm{CO}_{2}$ at $100 \%$ humidity using a Diaphot TMD (Nikon, Melville, NY) inverted microscope with a Nikon $40 \times$ Fluor oil immersion objective. A computer-controlled microscope stage (MLC-3, Märzhäuser Wetzlar GmbH, Wetzlar, Germany) permitted observation of up to 30 growth cones at different locations in a single dish during an experiment. Several hundred growth cones were examined during the course of this study. Both control (responding to the first stimulation; see Results) and experimental (responding to the second stimulation) growth cones could be monitored in the same dish. Images were acquired with a slow-scan cooled CCD camera (Photometrics, Tucson, AZ) at 5 min intervals and analyzed with Image 1.49 software (National Institutes of Health, Bethesda, MD). To be included in a data set, growth cones had to advance for at least $30 \mathrm{~min}$ before stimulation. Growth cones contacting other neurites or growth cones during the experiment were excluded from further analysis. In most cases at least three independent experiments were performed for a single data set. Both the number of growth cones and the number of experiments are included in Results.

Pharmacological treatments. For several experiments a specific inhibitor of the calcium-calmodulin protein kinase II (CaMKII), KN-93 (Sumi et al., 1991), or an inhibitor of protein kinase A (PKA), KT5720 (Kase et al., 1987), was added to the culture medium 30 min before positioning of the laminin-coated beads or onset of the electrical stimulation and was maintained in the medium for the duration of the experiment. With this procedure the effect of the inhibitor on the response to the priming stimulus could be tested also. KN-93 is known to prevent accelerated outgrowth after laminin bead contact (Kuhn et al., 1998), so its effect in the two-bead paradigm was not tested.

Quantification of filopodial activity. Filopodial distribution was determined by counting filopodia and measuring filopodial length on the left and right halves of the growth cone with IPLab Spectrum 3.1a software (Signal Analytics, Vienna, VA) on a Power Macintosh computer. A line along the medial longitudinal axis of the proximal neurite was extended through the growth cone to define left and right halves. Only filopodia that were $>2 \mu \mathrm{m}$ in length and that could be measured along their entire length (thereby excluding filopodia "waving" out of the plane of focus) were included in the data set. Total filopodial length was obtained by
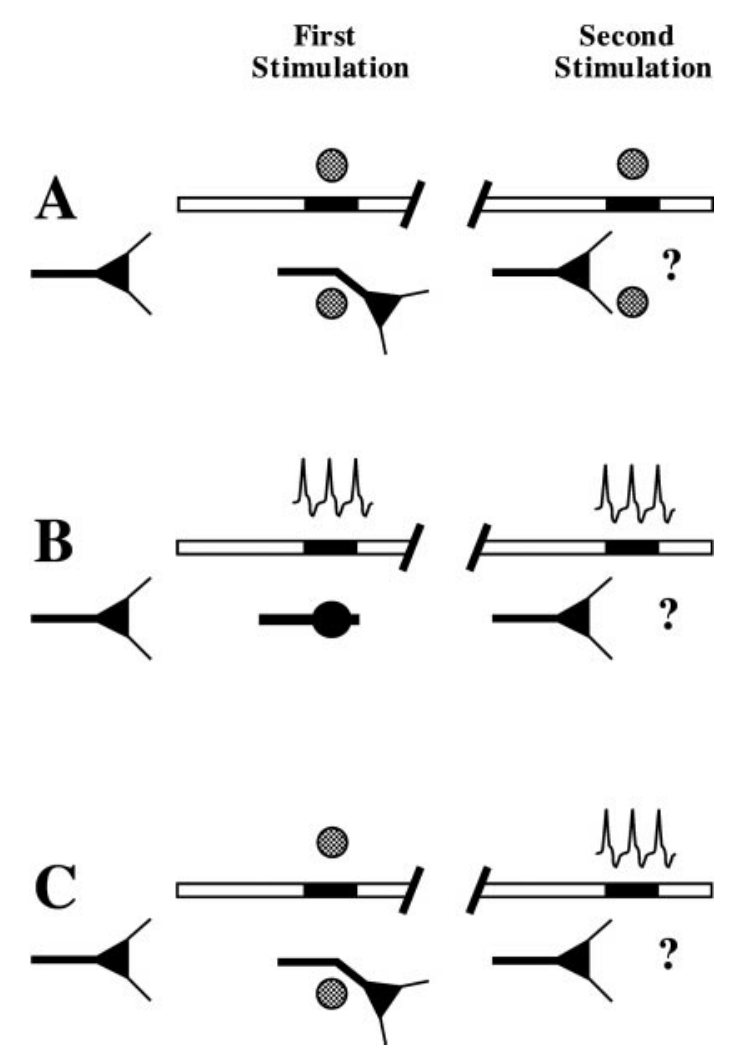

Figure 1. Schematic timeline of the three basic experimental paradigms used in this study. In the first set of experiments the growth cones were presented with two laminin-coated beads in sequence. The growth cones responded to the first laminin-coated bead with a turn and acceleration. In the second set of experiments the growth cones were stimulated electrically for two separate $10 \mathrm{~min}$ periods. The first stimulation typically resulted in growth cone collapse. In the third set of experiments the effect of dissimilar sequential stimuli was tested by presenting growth cones with a laminin-coated bead, followed by electrical stimulation. The response to the second stimulus was used to determine the effect of the previous first stimulation. In all instances the interval between the first and second stimulation could be varied.

summing the lengths of individual filopodia on both halves of the growth cone.

Statistical analysis. Comparison of quantitative measures used the two-sided Student's $t$ test. Comparison of response frequencies used the $\chi^{2}$ test. Microsoft Excel was used for these statistical tests.

\section{RESULTS}

The experiments described below provide a direct test of the hypothesis that previous exposure to a stimulus can alter the responses of growth cones to subsequent stimulation. Growth cones were subjected to sequential stimulation paradigms (Fig. 1) consisting of two similar or dissimilar stimuli presented at predetermined intervals. Using laminin-coated beads or electrical stimulation, we tested the effect of sequential stimuli in one of three combinations: sequential contact of two laminin-coated beads (Fig. 1A), two identical periods of electrical stimulation (Fig. $1 B$ ), or contact of a single bead followed by a single period of electrical stimulation (Fig. 1C).

\section{Growth cone responses to sequential encounters with laminin-coated beads}

Growth cones advancing on a fibronectin substrate display a stereotyped behavioral response to contact with a laminin-coated bead (Kuhn et al., 1995). The response consists of a turn toward 

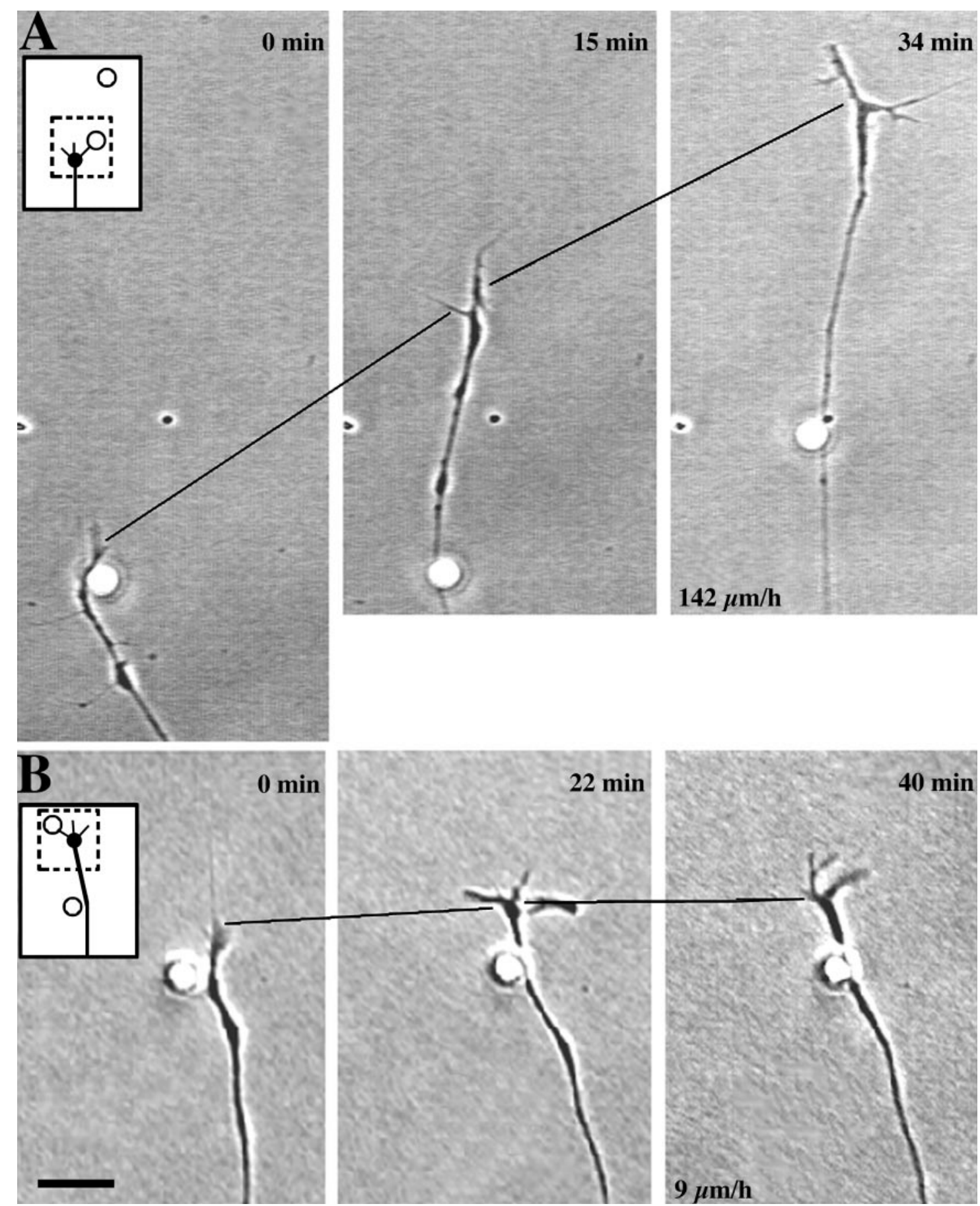

Figure 2. Phase-contrast images of growth cone encounters with laminin-coated beads. The stall behavior was seen only after a second laminin-coated bead encounter. Contact with a single laminin-coated bead resulted in the growth cone turning toward (at time 0 min) and extending past the bead (at time $15 \mathrm{~min}$ ). By $34 \mathrm{~min}$ the growth cone had advanced a distance of $81 \mu \mathrm{m}$ (pulling of the bead by the neurite had dislodged the bead by the $34 \mathrm{~min}$ image). A second encounter with a laminin-coated bead produced a growth cone stall. This growth cone encountered a second laminin bead at 0 min. By $22 \mathrm{~min}$ the growth cone had proceeded just past the bead. The growth cone failed to extend further even 40 min after bead contact. The total advance of the growth cone $(6 \mu \mathrm{m})$ was completed within $22 \mathrm{~min}$. Motile filopodia were observed during the entire period of observation. Scale bar, $10 \mu \mathrm{m}$.

the bead, a short pause near the bead, and, finally, rapid growth past the bead (Fig. 2A) (Kuhn et al., 1995). We tested whether a subsequent encounter with a second laminin-coated bead resulted in the same behavioral response.

The response to an encounter with a second laminin-coated bead differed both qualitatively and quantitatively from the response to a first laminin-coated bead. Of those growth cones that responded to and advanced past a first laminin-coated bead (62 of 62 in 25 independent experiments), all responded with turning to a second laminin-coated bead (48 of 48 in 21 independent experiments). However, after contacting a second laminin-coated bead, nearly one-half of these growth cones $(40 \%$; 19 of 48$)$ exhibited a novel, altered response never observed after encounter with a single laminin-coated bead: the growth cones ceased forward 


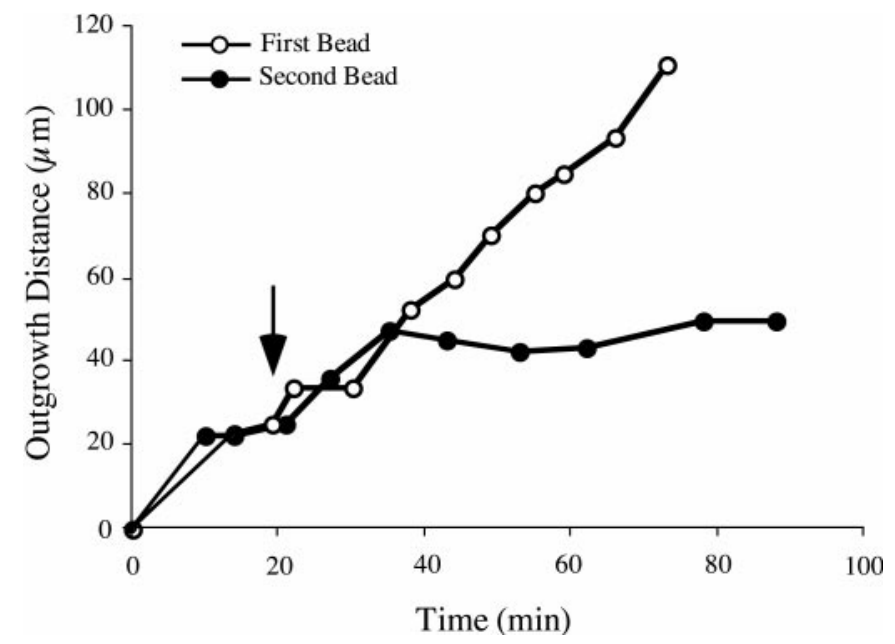

Figure 3. Growth cone stalls consisted of a dramatic reduction in outgrowth. The distance covered by a single growth cone is plotted as a function of time after contact (arrow) with the first laminin-coated bead (open circles) and later with the second laminin-coated bead (filled circles). After encountering the second bead, the growth cone advanced for $\sim 15$ min before ceasing forward advance (plateau) for the remainder of the observation period.

advance for the remainder of the experiment (up to $190 \mathrm{~min}$; Fig. $2 B$ ). This altered response, which we term a "stall," was entirely dependent on previous contact with a laminin-coated bead. Growth cones that stalled displayed only minor advance over a 1 hr period $(16.8 \pm 5.3 \mu \mathrm{m} ; n=14$; eight independent experiments) as compared with nonstalled counterparts $(88.9 \pm 9 \mu \mathrm{m} ; n=28$; 19 independent experiments) $(p<0.005)$. Much of the distance covered by growth cones before the stall occurred within the first 20 min after bead contact (Figs. 2B, 3). Stall behavior was never observed spontaneously, after contact with a single laminincoated bead, or after contact with a second BSA-coated bead $(n=$ 30; four independent experiments). Despite a lack of forward advance in stalled growth cones, and in contrast with the "collapsed" growth state, stalled growth cones displayed continual filopodial activity. The average total number of filopodia on stalled growth cones ( $3.4 \pm 0.3$ filopodia; $n=11$ growth cones) did not differ from the number of filopodia on growth cones both before $(4.5 \pm 0.6 ; n=12)$ and after $(3.6 \pm 0.3 ; n=12 ; p>0.05)$ contact with the first laminin-coated bead, nor were there any measurable left/right asymmetries in the number of filopodia. The summed lengths of filopodia also remained unchanged during the experiment (before first laminin-coated bead contact, $33.8 \pm$ $5.9 \mu \mathrm{m}, n=12$; after first laminin-coated bead contact, $26.2 \pm 2.7$ $\mu \mathrm{m}, n=12$; after second laminin-coated bead contact, $22.2 \pm 2.1$ $\mu \mathrm{m}, n=11 ; p>0.05)$. Thus, stall behavior was characterized as a lack of forward advance with filopodial activity indistinguishable from advancing growth cones.

Because different growth cones contacted the second laminincoated bead at different intervals after the first laminin bead, the incidence of stall behavior was examined as a function of the interval between laminin bead contacts (Fig. 4). At intervals $<30$ min, none of the growth cones displayed stall behavior ( 0 of 15$)$. For intervals between 31 and $60 \mathrm{~min}, 36 \%$ (5 of 14) of growth cones stalled. For intervals between 61 and $100 \mathrm{~min}, 74 \%$ (14 of 19) stalled. Thus, stall behavior increased with the interval between contacts and showed a response window in which a minimum interval was required before the stall behavior occurred.

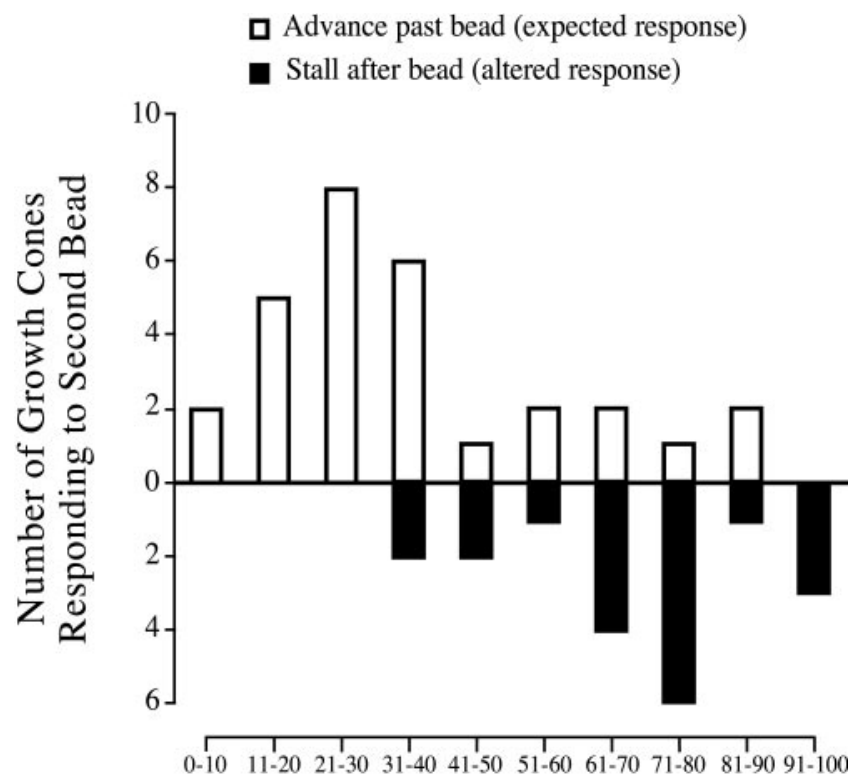

\section{Interval Between Bead Contacts (min)}

Figure 4. Growth cone responses to laminin-coated bead contacts depend on the interval between bead contacts. The number of growth cones responding to a second laminin-coated bead is shown as a function of the interval between the first and second bead contacts. At intervals $<30 \mathrm{~min}$ all of the growth cones showed the expected response of continued advance past the second bead (open bars). At intervals $>30$ min more growth cones displayed an altered response: stall. Data were acquired from 21 independent experiments (48 growth cones).

Stall responses were not related to the time of bead contact during the course of an experiment, because the proportion of growth cones stalling after a second laminin-coated bead did not change as a function of time under the microscope.

Together, these results show that growth cones can alter their responses in a history-dependent manner and that historydependent responses are dependent on the interval between laminin-coated bead contacts.

Growth cone responses to sequential electrical stimuli

In vitro studies demonstrate that depolarization and the associated action potential activity can inhibit neurite outgrowth by causing the collapse and retraction of growth cones (Cohan and Kater, 1986; Cohan et al., 1987; Cohan, 1990; Fields et al., 1990; Kater and Mills, 1991). We therefore tested whether electrical stimulation would alter the response of growth cones to a subsequent identical period of electrical stimulation.

Electrical stimulation was used to evoke action potentials in neurites [which subsequently propagated to the associated growth cones (Amato et al., 1996)] passing under the glass coverslip barrier in a modified Campenot chamber. Collapse was evident from the rapid withdrawal of filopodia and lamellipodia, frequently followed by an actual retraction of the growth cone (Fig. 5 ). Electrical stimulation-evoked growth cone collapse could be reduced significantly by the blockade of sodium channels with 50 nM tetrodotoxin (control, 82\% collapse; 90 of 110 in 24 independent experiments vs TTX, $20 \%$ collapse; 6 of 30 in two independent experiments).

The sequential electrical stimulation paradigm consisted of an initial $10 \mathrm{~min}$ stimulation period, followed by an interstimulus interval of a predetermined duration and a second 10 min stim- 

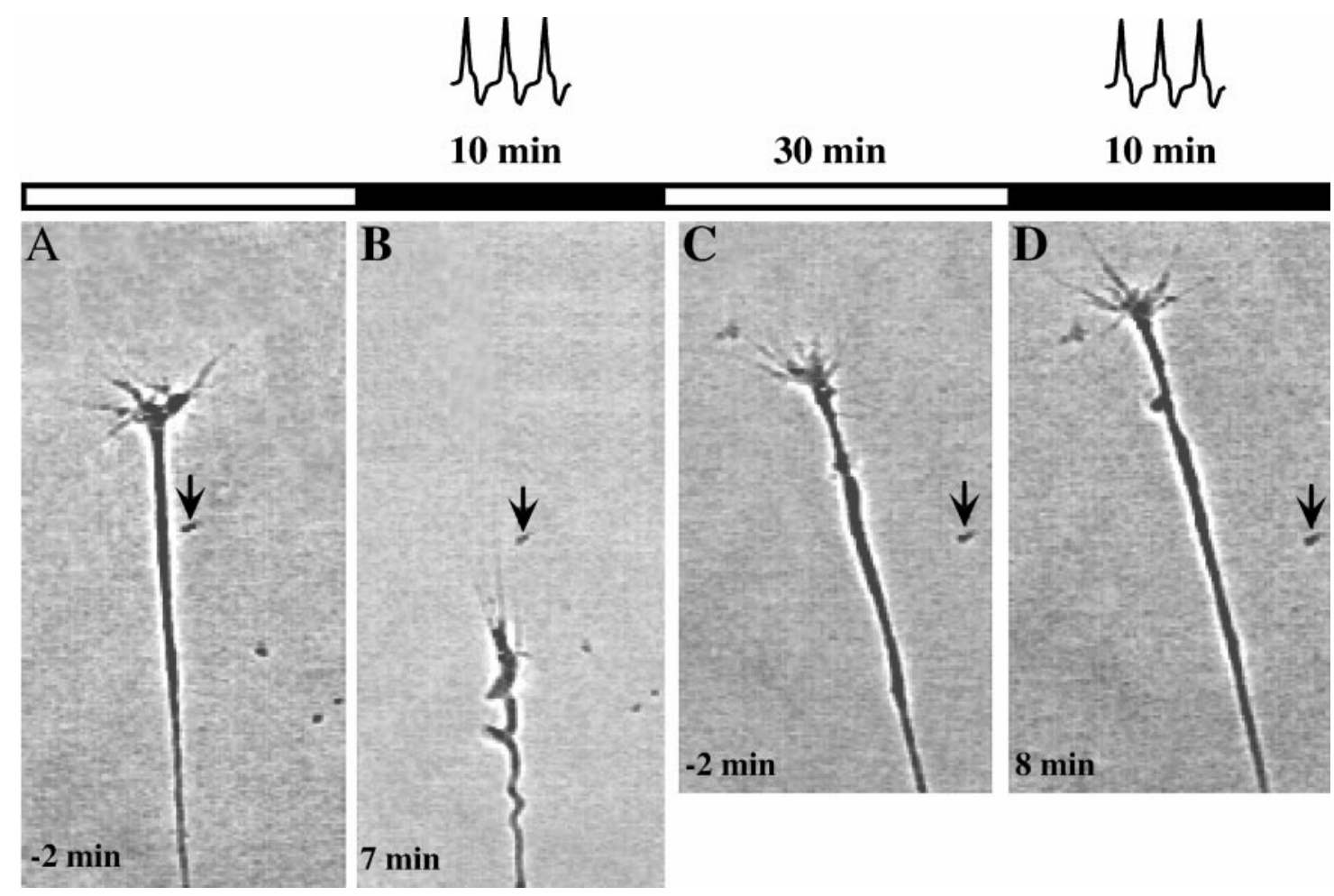

Figure 5. Growth cones respond differently to the first and second periods of electrical stimulation. Shown is a growth cone advancing normally 2 min before the onset of a first electrical stimulation. At $7 \mathrm{~min}$ after the onset of the first stimulation the growth cone showed typical collapse and retraction. The same growth cone subsequently recovered and resumed advance. This image was obtained 2 min before the second stimulation ( 30 min interstimulus interval). At 8 min after the onset of the second stimulation the growth cone continued advancing (which it did for at least an additional 36 min), with no visible response to the second stimulation. The arrow indicates the same fiduciary mark in the four panels. Scale bar, $10 \mu \mathrm{m}$. The microscope field was shifted slightly between images $B$ and $C$.

ulation period. The first stimulation period resulted in the expected response of growth cone collapse (82\%; 90 of 110) within 5 min after stimulus onset. The majority of the collapsed growth cones $(96 \%$; 86 of 90$)$ recovered within 15 min after the end of the first stimulation period (Fig. $5 C$ ). Only growth cones that recovered within $20 \mathrm{~min}$ after the initial stimulation were examined for their response to a second period of electrical stimulation

In contrast to the robust collapse resulting from an initial period of electrical stimulation, only one-third of the growth cones ( $35 \%$; 30 of 86 in 15 independent experiments) collapsed in response to a second, identical period of stimulation. The other two-thirds (65\%; 56 of 86) showed an altered response: they continued to advance up to $60 \mathrm{~min}$ after the second stimulation (Fig. 5D). Thus, a significant proportion of growth cones showed an altered response to a collapse-inducing stimulus after a previous stimulation.

The interval between the two stimulation periods determined the proportion of growth cones that showed an altered response to a second electrical stimulation. Growth cones stimulated at intervals $\leq 90 \mathrm{~min}$ displayed significantly fewer collapse responses (17\%; 9 of 52 in 11 independent experiments) (Fig. 6A) than growth cones stimulated at intervals $>90 \mathrm{~min}(62 \% ; 21$ of 34 in four independent experiments; $p<0.05$ ).

An alternative interpretation of these results could be that the stimulation efficacy became reduced significantly during the experiment because of the polarization of electrodes or another time-dependent factor. We were able to discount this possibility by using $\mathrm{KCl}$-induced depolarization as an alternative second stimulation. Growth cones were stimulated with an initial $10 \mathrm{~min}$ stimulation period as before, but instead of a second period of electrical stimulation the growth cones were depolarized with 60 $\mathrm{mm} \mathrm{KCl}$ as a second stimulus. Whereas most growth cones collapsed after a first electrical stimulation (85\%; 46 of 54 in six independent experiments) (Fig. 6B), only $11 \%$ (1 of 9) of the growth cones collapsed after $\mathrm{KCl}$ depolarization. This reduced response to $\mathrm{KCl}$ depolarization was not significantly different from the response to a second period of electrical stimulation (14\%; 4 of 28 in three independent experiments; $p>0.05) . \mathrm{KCl}$ depolarization in the absence of previous electrical stimulation resulted in a significantly higher probability of collapse $(62 \% ; 36$ of 58 in three independent experiments; $p<0.005)$. Thus, the failure to respond to a second electrical stimulation did not result from a change in stimulation efficacy during the experiment.

\section{Growth cone responses to dissimilar sequential stimuli}

A previous laminin-coated bead encounter or a previous electrical stimulation resulted in altered responses to a subsequent identical stimulus. To test whether this phenomenon can be generalized across dissimilar stimuli, we tested the effect of previous laminin-coated bead contact on the response of growth cones to electrical stimulation. Less than 30\% (29\%; 7 of 24 in three independent experiments) of growth cones that previously had encountered a laminin-coated bead responded to electrical stimulation with the expected collapse behavior (Fig. 7A). The probability of collapse was significantly less than the probability observed in control growth cones in the same dishes, which previously had not contacted a laminin-coated bead $(88 \% ; 7$ of 8 in three independent experiments; $p<0.005)$. Thus, previous 
A

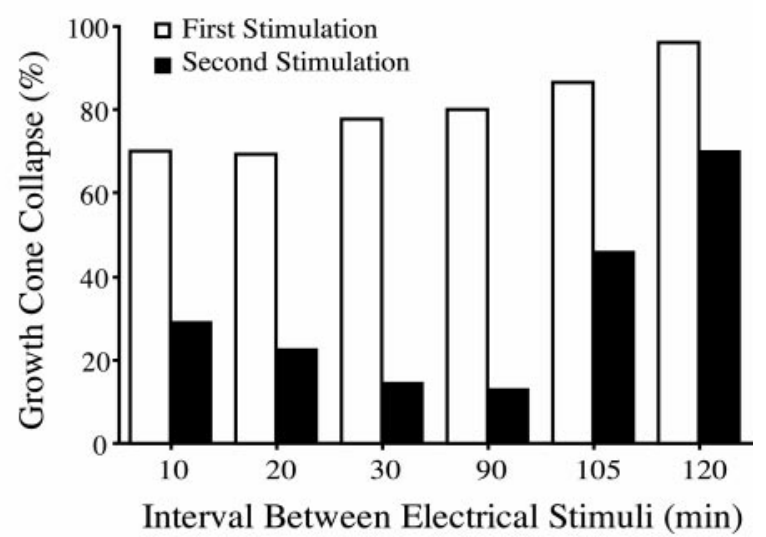

B

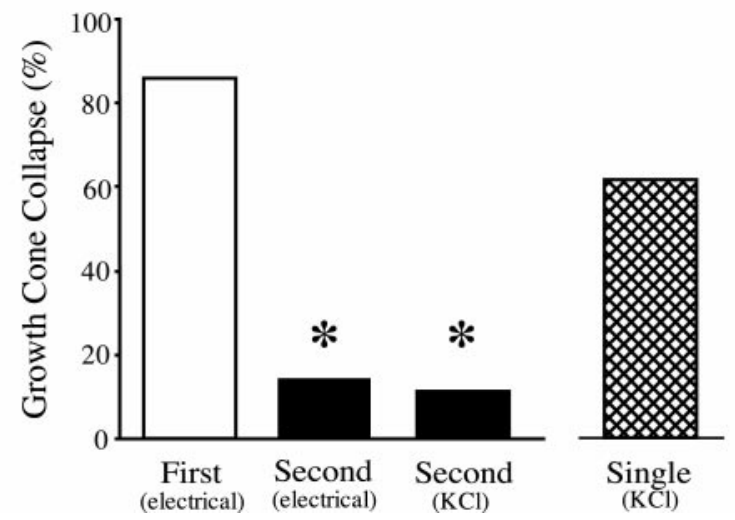

Figure 6. Growth cone response to electrical stimulation depends on the interval between stimulation periods. $A$, Growth cone collapse induced by electrical stimulation. Shown are the collapse after the first stimulus for each interval pair (open bars) and the collapse after the second stimulus of each interval pair ( filled bars). More growth cones failed to collapse in response to the second stimulus. This history-dependent suppression of collapse was a function of the interval between the two stimuli. Data were obtained from 15 independent experiments (86 growth cones). $B, \mathrm{KCl}$ used as the alternative second stimulus mimics the effect of the second electrical stimulation shown in $A . \mathrm{KCl}$ presented after an initial electrical stimulation $\left(\operatorname{Second}_{(\mathrm{KCl})}, 11 \% ; 1\right.$ of 9 in three independent experiments) produced a similar effect on growth cone responses as did a second electrical stimulation $\left(\right.$ Second $_{\text {(electrical) }}, 14 \%$; 4 of 28 in three independent experiments). In contrast, growth cones from the same dishes that did not receive a first electrical stimulation showed significantly greater collapse (62\%; 36 of 58 in three independent experiments) in response to the same $60 \mathrm{~mm} \mathrm{KCl}$ exposure $\left(\right.$ Single $\left._{(\mathrm{KCl})}\right)$. This incidence of collapse was less than that seen with first electrical stimulation (First (electrical), $85 \%$; 46 of 54 in six independent experiments). Stimuli were presented for $10 \mathrm{~min}$ with a $30 \mathrm{~min}$ interval between sequential presentations; ${ }^{*} p<0.005$.

contact with a laminin-coated bead in large part blocked the effect of electrical stimulation. Unlike the previous experiments, however, there was no correlation between the probability of collapse and the interval between laminin bead contact and electrical stimulation (Fig. 7B).

The reverse experiment (testing the effect of previous electrical stimulation on the response to laminin-coated beads) was not feasible. Experiments that used laminin-coated beads as a stimulus require a fibronectin substrate (Kuhn et al., 1995); growth cones extending on fibronectin do not recover from depolarizationinduced collapse (Diefenbach and Kater, 1998) and therefore would be unable to respond to a laminin-coated bead.
A
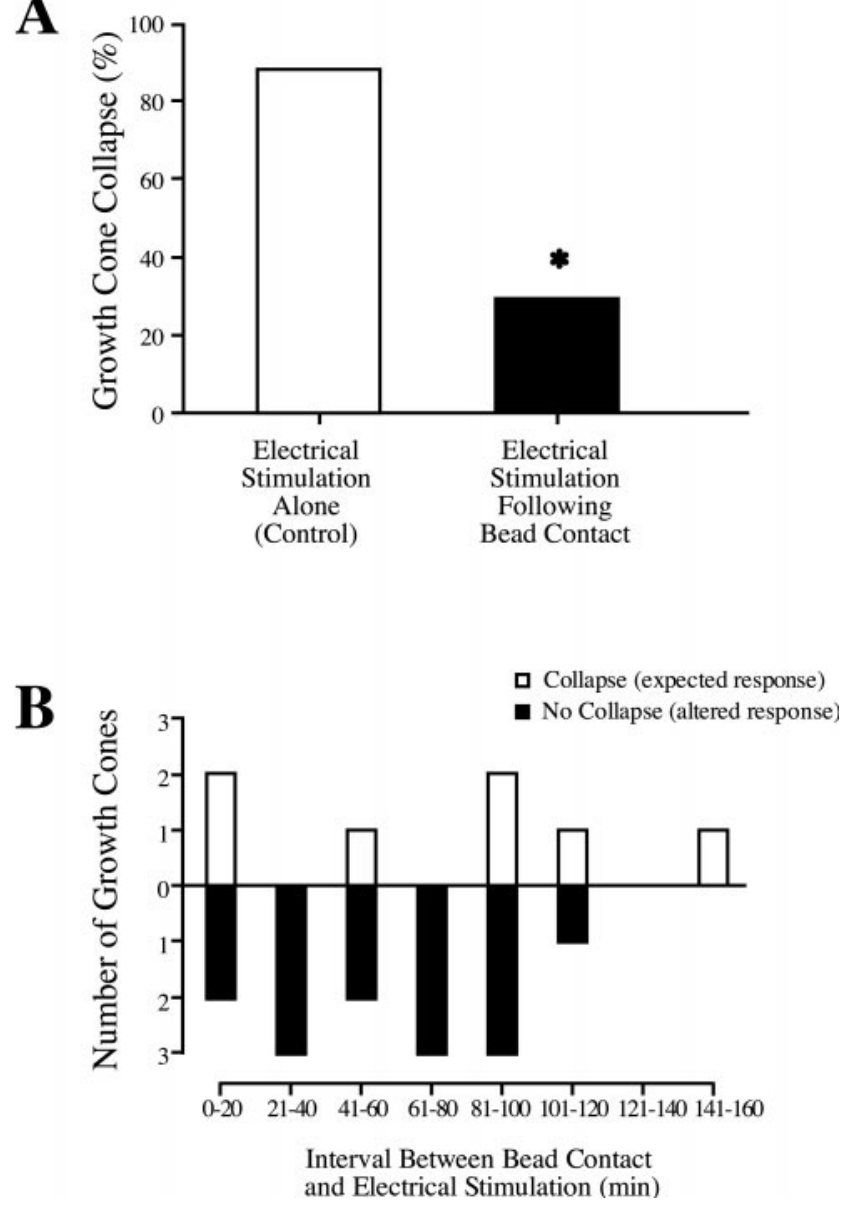

Figure 7. Previous contact with a laminin-coated bead decreases the probability of growth cone collapse in response to electrical stimulation. $A$, Less than $30 \%$ ( 7 of 24 in three independent experiments) of growth cones that previously contacted a laminin-coated bead collapsed in response to a 10 min electrical stimulation (Electrical Stimulation Following Bead Contact), whereas growth cones that had no previous encounter with a laminin-coated bead collapsed $88 \%$ ( 7 of 8 in three independent experiments) of the time in response to electrical stimulation (Electrical Stimulation Alone); ${ }^{*} p<0.05$. B, Growth cones that previously had contacted a laminin-coated bead fail to respond to electrical stimulation independent of the interval between stimuli. The number of growth cones responding to a 10 min electrical stimulation is shown as a function of the interval between laminin bead contact and electrical stimulation $(n=21$; three independent experiments). Open bars, The number of growth cones that showed the expected collapse response. Filled bars, The number of growth cones that continued outgrowth during and after electrical stimulation (altered response). Although it may appear that there are fewer growth cones showing the expected response between 20 and $80 \mathrm{~min}$, there was no significance in the relationship between the interstimulus interval and the growth cone response.

\section{A possible role for CaMKII in growth cone responses to sequential stimuli}

Both contact with a laminin substrate (Bixby et al., 1994; Kuhn et al., 1998) and electrical stimulation (Cohan et al., 1987; Lnenicka and Hong, 1997; Torreano and Cohan, 1997) cause an increase in calcium concentration in the growth cone. Indeed, the collapse response of growth cones to a single 10 min electrical stimulation was reduced from $82 \%$ (90 of 110) to $21 \%$ (13 of 62 ; three independent experiments) by the nonselective calcium channel blocker $\mathrm{NiCl}_{2}(500 \mu \mathrm{M} ; p<0.0001)$. A possible mediator of the effects of calcium influx is CaMKII, which has been shown to 
regulate growth cone motility and neurite outgrowth (Goshima et al., 1993; VanBerkum and Goodman, 1995; Williams et al., 1995; Nomura et al., 1997). In addition, CaMKII is known to be involved in the response of growth cones to laminin-coated beads (Kuhn et al., 1998). We therefore tested whether CaMKII activity is involved in mediating the effect of previous stimulation on growth cone responses. KN-93, a selective inhibitor of CaMKII (Sumi et al., 1991), was used in two of the three sequential stimulation paradigms: sequential electrical stimulation and laminin bead contact followed by electrical stimulation. It was not feasible to test the effect of KN-93 in the sequential laminincoated bead paradigm. CaMKII is required for rapid outgrowth after laminin-coated bead contact (Kuhn et al., 1998); thus, CaMKII inhibition would alter the response to a first laminincoated bead, making interpretation of the response to a second laminin-coated bead problematic.

The response to a second electrical stimulation (after a $30 \mathrm{~min}$ interstimulus interval) was affected significantly by KN-93. KN-93 $(2 \mu \mathrm{M})$ had no effect on the response of growth cones to a first stimulation (84\% collapse; 21 of 25 in two independent experiments) as compared with the response in the absence of KN-93 (78\%; 28 of 36 in six independent experiments; $p>0.05$ ) (Fig. $8 A$ ). The rate of advance of growth cones $30 \mathrm{~min}$ before KN-93 addition $(91.7 \pm 7.1 \mu \mathrm{m} / \mathrm{hr} ; n=24)$ was not significantly different from the rate of advance $30 \mathrm{~min}$ after KN-93 addition $(96.1 \pm 9.4 \mu \mathrm{m} / \mathrm{hr} ; n=25 ; p>0.050)$. In contrast, the percentage of growth cones collapsing after the second stimulation in the presence of $\mathrm{KN}-93$ (75\%; 12 of 16 in two independent experiments) was significantly greater than the response to a second stimulation in the absence of KN-93 (14\%; 4 of 28 in six independent experiments; $p<0.05$ ) (Fig. $8 A$ ). An intermediate concentration of $\mathrm{KN}-93(1.5 \mu \mathrm{M})$ produced an intermediate effect (40\%; 10 of 25 in six independent experiments).

cAMP has been shown to be important in reversing chemotactic responses of growth cones to trophic factors (Song et al., 1997, 1998). Inhibition of PKA with KT5720 (50 nM; Gadbois et al., 1992; Kuhn et al., 1995) failed to alter the effect of previous electrical stimulation on growth cone responses (KT5720, 0\% collapse, 0 of 7; control, 14\% collapse, 4 of 28 in two independent experiments; $p>0.05$ ). Furthermore, growth cone responses to the first electrical stimulation were unaffected by KT5720 treatment (69\% collapse, 9 of 13 in two independent experiments) when compared with controls stimulated in the absence of KT5720 (78\% collapse, 29 of 37; $p>0.05)$.

We also tested whether CaMKII inhibition could alter the response to a subsequent electrical stimulation when the first stimulus was contact with a laminin-coated bead. Indeed, growth cones that previously had responded to a laminin bead had a much higher probability of collapsing in response to electrical stimulation in the presence of $2.0 \mu \mathrm{M} \mathrm{KN}-93$ (83\%; 10 of 12 in three independent experiments) (Fig. $8 B$ ) than in the absence of KN-93 (29\%; 7 of 24 in three independent experiments; $p<$ $0.005)$. This response to electrical stimulation in the presence of $\mathrm{KN}-93$ was not significantly different from the overall response to a single period of electrical stimulation $(82 \%$; 90 of $110 ; p>$ $0.05)$. These results, along with the results from the sequential electrical stimulation paradigm, implicate CaMKII in mediating history-dependent changes in growth cone responses.

\section{DISCUSSION}

The present results support a view of growth cone guidance in which the response to a given stimulus is not absolutely stereo-
Control (single electrical stimulation)

Second (electrical followed by electrical)

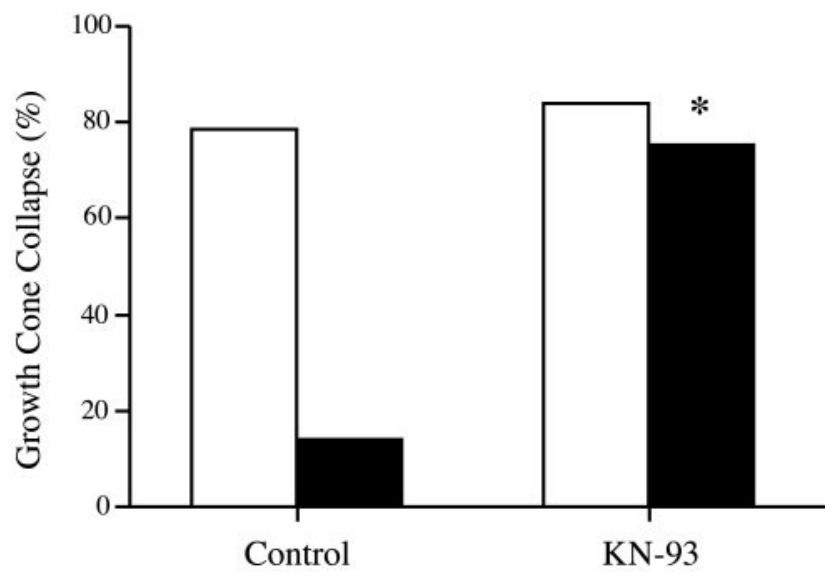

B

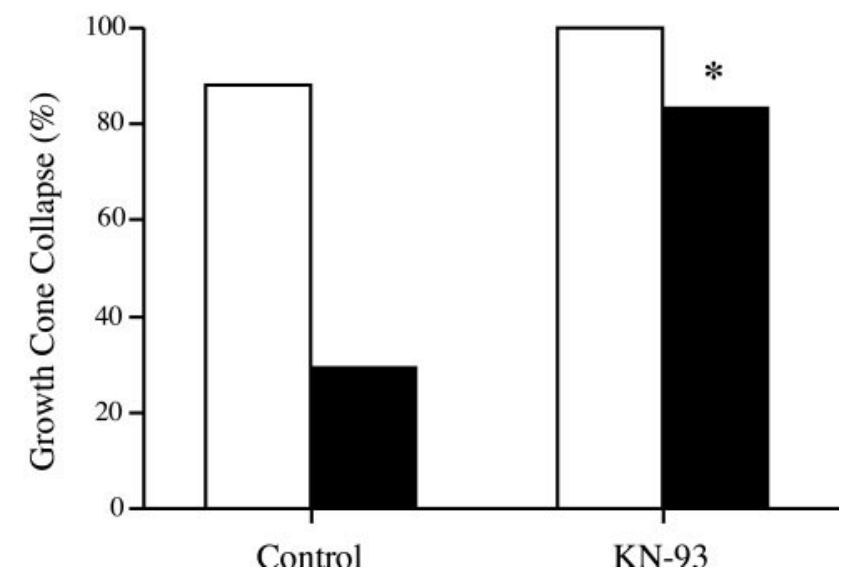

Figure 8. A possible role for CaMKII in history-dependent responses of growth cones. KN-93 $(2.0 \mu \mathrm{M})$ was added $30 \mathrm{~min}$ before the first stimulation and remained in the bath for the rest of the experiment. $A$, Sequential electrical stimulation. Whereas only $14 \%$ of growth cones collapsed after a second stimulation in the absence of KN-93 ( filled bar, Control; 4 of 28 in six independent experiments), $75 \%$ of growth cones collapsed in the continued presence of KN-93 ( filled bar, KN-93; 12 of 16 in two independent experiments; $\left.{ }^{*} p<0.05\right)$. KN-93 did not alter the normal response to the first stimulation (open bar, KN-93; 84\% collapse, 21 of 25 in two independent experiments). $B$, An effect of previous laminin-coated bead contact on the response to subsequent electrical stimulation. In the absence of KN-93, few growth cones collapsed if they previously encountered a laminin-coated bead ( filled bar, Control; 29\%; 7 of 24 in three independent experiments). In contrast, $\mathrm{KN}-93$ treatment resulted in a greater number of growth cones collapsing in response to the electrical stimulation ( filled bar, $\mathrm{KN}-93 ; 83 \%$; 10 of $12 ; * p<0.005$ ). Blocking the CaMKII pathway thus appears to negate the effect of previous laminin bead contact. KN-93 treatment had no affect on the response of growth cones to a single electrical stimulation (open bar, KN-93; 100\%; 5 of 5).

typed but rather is dependent on previous stimulation, that is, the stimulus history of the growth cone. Growth cones responded to an initial laminin-coated bead contact with the expected turn and acceleration previously reported; the response to a subsequent 
bead contact, however, frequently elicited the stall behavior. Growth cones that had recovered from an electrical stimulationinduced collapse became resistant to a subsequent identical period of electrical stimulation. Finally, growth cones became resistant to the collapse-inducing effects of electrical stimulation after contact with a laminin-coated bead.

The idea that growth cone responses are history-dependent, as demonstrated in the present study, is supported by a small set of reports in the literature. Earlier studies made the incidental observation that, whereas growth cones would collapse on an initial encounter with a neurite, growth cones eventually traversed the neurites after subsequent encounters (Kapf hammer et al., 1986; Honig and Burden, 1993). In addition, retinal ganglion cell growth cones have been shown to grow onto a normally inhibitory substrate of chondroitin sulfate proteoglycan (CSPG) when presented as a step gradient of increasing concentration (Snow and Letourneau, 1992). Thus, a previous step of lower concentration "adapted" a small percentage of growth cones over a period of hours, enabling them to proceed onto a step on higher concentration of CSPG. In a recent in situ study, commissural axons were found to lose their responsiveness to a floor plate explant (a source for a chemotaxic guidance cue) after a previous floor plate crossing (Shirasaki et al., 1998). When taken together with the present study, these findings suggest that growth cones in other systems can show altered responses to specific stimuli resulting from a previous stimulus.

\section{Relationship between growth cone response and interstimulus interval}

The specific response of a growth cone to contact with a second laminin-coated bead was dictated, in part, by the interval between bead contacts. Intervals shorter than 30 min resulted in continued elongation, whereas intervals $>30$ min usually resulted in a stall. In our earlier report (Kuhn et al., 1995) growth cones encountering laminin-coated beads showed rapid outgrowth between beads contacted in sequence; however, the effect of longer intervals between bead contacts was not tested. In fact, it should be emphasized that there have been few attempts in the literature to examine the behavior of individual growth cones at intervals as long as described here, and, accordingly, earlier work would not have observed such stall events. In the present study the growth cones showed an altered behavioral response; this altered response was observed only after a minimum interstimulus interval of 30 min and was never observed after an initial bead contact. It is possible that contact with a laminin-coated bead initiates a transient $(\sim 30 \mathrm{~min})$ transduction process in the growth cone that must be completed for a second bead contact to result in expression of the stall behavior. When bead contacts occur over a shorter interval, the transduction process might be "retriggered" before it has become complete.

The response of growth cones to a second period of electrical stimulation was also dependent on the interval between stimulus periods. Interstimulus intervals of $<90 \mathrm{~min}$ resulted in an altered response: continued outgrowth instead of collapse. In contrast to the sequential laminin-coated bead experiments, however, this change in growth cone response developed as rapidly as could be tested and became less likely with longer interstimulus intervals. It is possible that electrical stimulation initiates a transient transduction process in the growth cone that results in an altered response to electrical stimulation. When this process is completed, the response of the growth cone to electrical stimulation then might revert to normal.
The altered response of growth cones to laminin bead contact only occurred when the interval between bead contacts was $>30$ min, whereas the altered response of growth cones to repeated electrical stimulation was observed with intervals as short as 10 min and decreased for intervals $>90 \mathrm{~min}$. This apparent difference in time course might suggest that different second messenger systems are involved. However, the timing differences are not as large as they might first appear. The minimum 30 min interbead interval represents the interval between the initial filopodial contact of the growth cone with the first and second laminincoated beads. During this interval the growth cone spends time extending toward, around, and past the first bead, so the time at which the relevant transduction process is initiated cannot be defined precisely. The electrical stimulation period lasts $10 \mathrm{~min}$; the total time between the onset of the first and second stimulus (for a 10 min interstimulus interval) therefore was $20 \mathrm{~min}$; furthermore, the effects of previous electrical stimulation appeared to be maximal with interstimulus intervals of at least $30 \mathrm{~min}$.

Differences in stimulation form and in culture conditions further complicate a detailed comparison of the time courses of the altered responses. It is quite likely that electrical stimulation is a more intense form of stimulation than contact with a laminincoated bead; a difference in the intensity of stimulation might result in a different time course of second messenger activation. In addition, neurons had to be cultured on a fibronectin substrate for the laminin-coated bead experiments. Growth cones show substrate-dependent differences in response to electrical stimulation as well as differences in the clearance of intracellular calcium (Diefenbach and Kater, 1998; our unpublished observations).

Thus, although there were differences in the time course of the effects of stimulus history in stimulation paradigms, they are not so dissimilar as to discount the operation of similar second messenger systems. Furthermore, because previous contact with a laminin-coated bead altered growth cone responses to electrical stimulation, there are likely to be shared steps in the signal transduction pathway mediating changes in responses to electrical stimulation and laminin bead contact.

\section{Calcium and CaMKII as potential players in the pathway leading to history dependence}

Laminin-coated beads and electrical stimulation are both known to cause transient increases in intracellular free calcium concentration (Cohan et al., 1987; Kuhn et al., 1998). Such changes in growth cone calcium are known to affect growth cone responses to various forms of stimulation (Kater and Mills, 1991). It is therefore possible that the altered response of growth cones to a second stimulation may result from these calcium transients. However, because of the relatively brief nature of calcium responses (in sec) relative to the much longer duration changes in growth cone responses (tens of minutes), we examined a downstream effector of calcium transients: the CaMKII.

CaMKII is known to be important in plasticity-associated changes in synaptic activity and morphology (Wu and Betz, 1996; Lisman et al., 1997). CaMKII is activated by elevated intracellular calcium levels; the activation can persist long after the calcium levels have returned to normal (Ishida et al., 1996; Putkey and Waxham, 1996; Giese et al., 1998). Kuhn and coworkers (1998) observed that, after extending past a laminin bead, growth cones displayed a delayed (28 min after initial laminin bead contact) and sustained increase in intracellular calcium that coincided with an increase in the extension rate of the growth cones. Furthermore, both the elevation in calcium and the increase in 
growth rate were blocked by the inhibition of CaMKII. In the present study the growth cones required at least $30 \mathrm{~min}$ before contacting a second laminin bead for stall behavior to be observed, and altered responses to a second electrical stimulation appeared to peak by $30 \mathrm{~min}$ after initial stimulation. Furthermore, the inhibition of CaMKII prevented the effect of previous stimulation in altering growth cone responses in the two experimental paradigms tested. This result implicates CaMKII as a candidate mediator of the effects of stimulus history on growth cone responses. There is a variety of potential downstream targets of CaMKII activation that could underlie the observed changes in growth cone responses, including the ryanodine receptor (Witcher et al., 1992), neurofilament proteins (Sihag and Nixon, 1989), the microtubule-associated protein, tau (Singh et al., 1996), and the CaM-dependent protein phosphatase, calcineurin (Hashimoto and Soderling, 1989).

\section{Growth cone stall behavior}

The stall behavior reported here consists of a long-term (at least $190 \mathrm{~min}$ ) cessation of growth cone extension without collapse and with persistent filopodial activity. In vivo, some growth cones display a stepwise form of advance (Bentley and Caudy, 1983; Palka et al., 1992; Godement et al., 1994; Mason and Wang, 1997) that can include pauses in outgrowth that can occur in "decision regions," regions where a growth cone is faced with alternative courses of advance (Tosney and Landmesser, 1985; Sretavan and Reichardt, 1993; Mason and Wang, 1997; Melancon et al., 1997). Pauses in growth cone extension also can occur during "waiting periods" that have been reported to last anywhere from 45 min to $48 \mathrm{hr}$ or longer (Tolbert et al., 1984; O'Leary and Terashima, 1988; Moody et al., 1989; Stainier and Gilbert, 1990; Ghosh and Shatz, 1992; Halloran and Kalil, 1994; Yamamoto et al., 1997). It is possible that the stall behavior observed in the present study is similar to the pause observed in vivo. Indeed, in a recent notable paper the growth cones showed pauses in outgrowth for up to 1 hr in vivo. Interestingly, spontaneous calcium transients occurred when growth cones reached positions along the outgrowth pathway where pauses normally would occur (Gomez and Spitzer, 1999). Questions for future study include how stall behavior is regulated, whether outgrowth can be reinitiated after a stall, and what factors may reinitiate outgrowth.

\section{Significance of stimulus history for growth cone guidance in vivo}

Growth cones navigate to their targets through a complex molecular terrain. In principle, specific guidance cues could be organized in a variety of forms ranging from discrete patches (stepping stones) to a continuous corridor of information. Furthermore, in the context of the present investigation an ascending or descending gradient distribution of a cue has interesting ramifications.

Discrete points of guidance information have been described for several in vivo systems. For example, laminin appears in a punctile distribution in parts of the CNS (Liesi, 1985; Liesi and Risteli, 1989; Zhou, 1990) in a pattern that can be considered similar to the sequential presentation of laminin-coated beads that were used in the present study. In the developing grasshopper limb bud, guidance cues also appear to be organized in a discrete stepping stone-like manner (Bentley and Caudy, 1983; Caudy and Bentley, 1986). There also is good evidence for gradients (both chemoattractant and chemorepellant) guiding growth cones both in vivo and in vitro (Baier and Bonhoeffer, 1992; Sato et al., 1994;
Colamarino and Tessier-Lavigne, 1995; Ebens et al., 1996; de la Torre et al., 1997; Metin et al., 1997; Richards et al., 1997; Bagnard et al., 1998; Rosentreter et al., 1998).

Based on the present findings, a growth cone encountering a stepping stone-like sequence of guidance cues in vivo may recognize each individual cue as novel if that cue is encountered with a sufficient delay after a previous cue; i.e., there would be no effect of stimulus history on the response of the growth cone. Alternatively, there may well be examples in vivo in which potential cues are arranged closely enough that, in fact, a growth cone can ignore this potential path in favor of novel information in the range of its far-reaching filopodia.

Similarly, gradients of information may well be arrayed so that they are either ignored or alternatively emphasized. If a growth cone followed the general scheme of history-dependent alteration of its responses, then a gradient could, in fact, be confounding and not guiding. Here again it may be that the interval between stimuli may be important. If new stimuli are encountered rapidly enough, as in a gradient, then the distinction necessary to acquire a "past" may not exist. Alternatively, recent ideas that growth cones do measure differences in space when being guided by a gradient (Rosentreter et al., 1998) alert us to the possibility that the question of history dependence may need to be defined specifically in each context in which there is growth cone pathfinding.

\section{Conclusions}

The results described here suggest that stimulus history may be an important factor for determining the relationship between information provided by an individual stimulus and the resultant response of a growth cone. A role for stimulus history was shown by using two very different stimuli: electrical stimulation and laminin-coated bead contact. In principle, the findings reported here might well be generalized to encompass many classes of guidance cues relevant to growth cone pathfinding. In this way the immediate local environment that previously was thought to be the primary determinant of growth cone behavior may become only one part of a guidance equation that relies on the precise and cumulative contributions of previous cues.

\section{REFERENCES}

Amato A, Al-Mohanna FA, Bolsover S (1996) Spatial organization of calcium dynamics in growth cones of sensory neurones. Dev Brain Res 92:101-110.

Bagnard D, Lohrum M, Uziel D, Puschel AW, Bolz J (1998) Semaphorins act as attractive and repulsive guidance signals during the development of cortical projections. Development 125:5043-5053.

Baier H, Bonhoeffer F (1992) Axon guidance by gradients of a targetderived component. Science 255:472-475.

Bentley D, Caudy M (1983) Pioneer axons lose directed growth after selective killing of guidepost cells. Nature 304:62-65.

Bixby JL, Grunwald GB, Bookman RJ (1994) $\mathrm{Ca}^{2+}$ influx and neurite growth in response to purified N-cadherin and laminin. J Cell Biol 127:1461-1475.

Block SM (1992) Making light work with optical tweezers. Nature 360:493-495.

Campenot RB (1977) Local control of neurite development by nerve growth factor. Proc Natl Acad Sci USA 74:4516-4519.

Caudy M, Bentley D (1986) Pioneer growth cone steering along a series of neuronal and non-neuronal cues of different affinities. J Neurosci 6:364-379.

Cohan CS (1990) Frequency-dependent and cell-specific effects of electrical activity on growth cone movements of cultured Helisoma neurons. J Neurobiol 21:400-413.

Cohan CS, Kater SB (1986) Suppression of neurite elongation and growth cone motility by electrical activity. Science 232:1638-1640.

Cohan CS, Connor JA, Kater SB (1987) Electrically and chemically 
mediated increases in intracellular calcium in neuronal growth cones. J Neurosci 7:3588-3599.

Colamarino SA, Tessier-Lavigne M (1995) The role of the floor plate in axon guidance. Annu Rev Neurosci 18:497-529.

Davenport RW, Thies E, Nelson PG (1996) Cellular localization of guidance cues in the establishment of retinotectal topography. J Neurosci 16:2074-2085.

de la Torre JR, Hopker VH, Ming GL, Poo M-M, Tessier-Lavigne M, Hemmati-Brivanlou A, Holt CE (1997) Turning of retinal growth cones in a netrin-1 gradient mediated by the netrin receptor DCC. Neuron 19:1211-1224.

Diefenbach TJ, Kater SB (1998) Substrate-dependent responses of neuronal growth cones to collapse-inducing stimulation. Soc Neurosci Abstr 24:29.

Ebens A, Brose K, Leonardo ED, Hanson MGJ, Bladt F, Birchmeier C, Barres BA, Tessier-Lavigne M (1996) Hepatocyte growth factor/scatter factor is an axonal chemoattractant and a neurotrophic factor for spinal motor neurons. Neuron 17:1157-1172.

Fields RD, Neale EA, Nelson PG (1990) Effects of patterned electrical activity on neurite outgrowth from mouse sensory neurons. J Neurosci 10:2950-2964.

Gadbois DM, Crissman HA, Tobey RA, Bradbury EM (1992) Multiple kinase arrest points in the G1 phase of nontransformed mammalian cells are absent in transformed cells. Proc Natl Acad Sci USA 89:86268630.

Ghosh A, Shatz CJ (1992) Pathfinding and target selection by developing geniculocortical axons. J Neurosci 12:39-55.

Giese KP, Fedorov NB, Filipkowski RK, Silva AJ (1998) Autophosphorylation at Thr286 of the alpha calcium-calmodulin kinase II in LTP and learning. Science 279:870-873.

Godement P, Wang L-C, Mason CA (1994) Retinal axon divergence in the optic chiasm: dynamics of growth cone behavior at the midline. J Neurosci 14:7024-7039.

Gomez TM, Spitzer NC (1999) In vivo regulation of axon extension and pathfinding by growth cone calcium transients. Nature 397:350-355.

Goshima Y, Ohsako S, Yamauchi T (1993) Overexpression of $\mathrm{Ca}^{2+}$ / calmodulin-dependent protein kinase II in Neuro2a and NG108-15 neuroblastoma cell lines promotes neurite outgrowth and growth cone motility. J Neurosci 13:559-675.

Halloran MC, Kalil K (1994) Dynamic behaviors of growth cones extending in the corpus callosum of living cortical brain slices observed with video microscopy. J Neurosci 14:2161-2177.

Hashimoto Y, Soderling TR (1989) Regulation of calcineurin by phosphorylation. Identification of the regulatory site phosphorylated by $\mathrm{Ca}^{2+} /$ calmodulin-dependent protein kinase II and protein kinase C. J Biol Chem 264:16524-16529.

Honig MG, Burden SM (1993) Growth cones respond in diverse ways upon encountering neurites in cultures of chick dorsal root ganglia. Dev Biol 156:454-472.

Isbister CM, Tsai A, Wong ST, Kolodkin AL, O’Connor TP (1999) Discrete roles for secreted and transmembrane semaphorins in neuronal growth cone guidance in vivo. Development 126:2007-2019.

Ishida A, Kitani T, Fujisawa H (1996) Evidence that autophosphorylation at Thr-286/Thr-287 is required for full activation of calmodulindependent protein kinase II. Biochim Biophys Acta 1311:211-217.

Kapfhammer JP, Grunewald BE, Raper JA (1986) The selective inhibition of growth cone extension by specific neurites in culture. J Neurosci 6:2527-2534.

Karlstrom RO, Trowe T, Klostermann S, Baier H, Brand M, Crawford AD, Grunewald B, Haffter P, Hoffmann H, Meyer SU, Muller BK, Richter S, van Eeden FJ, Nusslein-Volhard C, Bonhoeffer F (1996) Zebrafish mutations affecting retinotectal axon pathfinding. Development 123:427-438.

Kase H, Iwahashi K, Nakanishi S, Matsuda Y, Yamada K, Takahashi M, Murakata C, Sato A, Kaneko M (1987) K-252 compounds, novel and potent inhibitors of protein kinase $\mathrm{C}$, and cyclic nucleotide-dependent protein kinases. Biochem Biophys Res Commun 142:436-440.

Kater SB, Mills LR (1991) Regulation of growth cone behavior by calcium. J Neurosci 11:891-899.

Kuhn TB, Schmidt MF, Kater SB (1995) Laminin and fibronectin guideposts signal sustained but opposite effects to passing growth cones. Neuron 14:275-285.

Kuhn TB, Williams CV, Dou P, Kater SB (1998) Laminin directs growth cone navigation via two temporally and functionally distinct calcium signals. J Neurosci 18:184-194.
Liesi P (1985) Do neurons in the vertebrate CNS migrate on laminin? EMBO J 4:1163-1170.

Liesi P, Risteli L (1989) Glial cells of mammalian brain produce a variant form of laminin. Exp Neurol 105:86-92.

Lisman J, Malenka RC, Nicoll RA, Malinow R (1997) Learning mechanisms: the case for CaM-KII. Science 276:2001-2002.

Lnenicka GA, Hong SJ (1997) Activity-dependent changes in voltagedependent calcium currents and transmitter release. Mol Neurobiol 14:37-66.

Mason CA, Wang LC (1997) Growth cone form is behavior-specific and, consequently, position-specific along the retinal axon pathway. J Neurosci 17:1086-1100.

Melancon E, Liu DW, Westerfield M, Eisen JS (1997) Pathfinding by identified zebrafish motoneurons in the absence of muscle pioneers. J Neurosci 17:7796-7804.

Metin C, Deleglise D, Serafini T, Kennedy TE, Tessier-Lavigne M (1997) A role for netrin-1 in the guidance of cortical efferents. Development 124:5063-5074.

Moody SA, Quigg MS, Frankfurter A (1989) Development of the peripheral trigeminal system in the chick revealed by an isotype-specific anti- $\beta$-tubulin monoclonal antibody. J Comp Neurol 279:567-580.

Nomura T, Kumatoriya K, Yoshimura Y, Yamauchi T (1997) Overexpression of alpha and beta isoforms of $\mathrm{Ca}^{2+} /$ calmodulin-dependent protein kinase II in neuroblastoma cells- $\mathrm{H}-7$ promotes neurite outgrowth. Brain Res 766:129-141.

O'Leary DDM, Terashima T (1988) Cortical axons branch to multiple subcortical targets by interstitial axon budding: implications for target recognition and "waiting periods." Neuron 1:901-910.

Palka J, Whitlock KE, Murray MA (1992) Guidepost cells. Curr Opin Neurobiol 2:48-54.

Putkey JA, Waxham MN (1996) A peptide model for calmodulin trapping by calcium/calmodulin-dependent protein kinase II. J Biol Chem 271:29619-29623.

Richards LJ, Koester SE, Tuttle R, O'Leary DD (1997) Directed growth of early cortical axons is influenced by a chemoattractant released from an intermediate target. J Neurosci 17:2445-2458.

Rosentreter SM, Davenport RW, Loschinger J, Huf J, Jung J, Bonhoeffer F (1998) Response of retinal ganglion cell axons to striped linear gradients of repellent guidance molecules. J Neurobiol 37:541-562.

Sato M, Lopez-Mascaraque L, Heffner CD, O'Leary DDM (1994) Action of a diffusible target-derived chemoattractant on cortical axon branch induction and directed growth. Neuron 13:791-803.

Shirasaki R, Katsumata R, Murakami F (1998) Change in chemoattractant responsiveness of developing axons at an intermediate target. Science 279:105-107.

Sihag RK, Nixon RA (1989) In vivo phosphorylation of distinct domains of the 70-kilodalton neurofilament subunit involves different protein kinases. J Biol Chem 264:457-464.

Singer MA, O'Connor TP, Bentley D (1995) Pioneer growth cone migration in register with orthogonal epithelial domains in the grasshopper limb bud. Int J Dev Biol 39:965-973.

Singh TJ, Wang JZ, Novak M, Kontzekova E, Grundke-Iqbal I, Iqbal K (1996) Calcium/calmodulin-dependent protein kinase II phosphorylates tau at Ser-262 but only partially inhibits its binding to microtubules. FEBS Lett 387:145-148.

Snow DM, Letourneau PC (1992) Neurite outgrowth on a step gradient of chondroitin sulfate proteoglycan (CS-PG). J Neurobiol 23:322-336.

Song HJ, Ming GL, Poo M-M (1997) cAMP-induced switching in turning direction of nerve growth cones. Nature 388:275-279.

Song HJ, Ming G, He Z, Lehmann M, McKerracher L, Tessier-Lavigne M, Poo M-M (1998) Conversion of neuronal growth cone responses from repulsion to attraction by cyclic nucleotides. Science 281:1515-1518.

Sretavan DW, Reichardt LF (1993) Time-lapse video analysis of retinal ganglion cell axon pathfinding at the mammalian optic chiasm: growth cone guidance using intrinsic chiasm cues. Neuron 10:761-777.

Stainier DY, Gilbert W (1990) Pioneer neurons in the mouse trigeminal sensory system. Proc Natl Acad Sci USA 87:923-927.

Stoeckli ET, Kuhn TB, Duc CO, Ruegg MA, Sonderegger P (1991) The axonally secreted protein axonin-1 is a potent substratum for neurite growth. J Cell Biol 112:449-455.

Sumi M, Kiuchi K, Ishikawa T, Ishii A, Hagiwara M, Nagatsu T, Hidaka 
H (1991) The newly synthesized selective $\mathrm{Ca}^{2+} /$ calmodulindependent protein kinase II inhibitor KN-93 reduces dopamine contents in PC12h cells. Biochem Biophys Res Commun 181:968-975.

Tolbert DL, Dunn RCJ, Vogler GA (1984) The postnatal development of corticotrigeminal projections in the cat. J Comp Neurol 228:478-490.

Torreano PJ, Cohan CS (1997) Electrically induced changes in $\mathrm{Ca}^{2+}$ in Helisoma neurons: regional and neuron-specific differences and implications for neurite outgrowth. J Neurobiol 32:150-162.

Tosney KW, Landmesser LT (1985) Growth cone morphology and trajectory in the lumbosacral region of the chick embryo. J Neurosci 5:2345-2358.

VanBerkum MFA, Goodman CS (1995) Targeted disruption of $\mathrm{Ca}^{2+}$ calmodulin signaling in Drosophila growth cones leads to stalls in axon extension and errors in axon guidance. Neuron 14:43-56.
Williams EJ, Mittal B, Walsh FS, Doherty P (1995) $\mathrm{A} \mathrm{Ca}^{2+} /$ calmodulin kinase inhibitor, KN-62, inhibits neurite outgrowth stimulated by CaMs and FGF. Mol Cell Neurosci 6:69-79.

Witcher DR, Strifler BA, Jones LR (1992) Cardiac-specific phosphorylation site for multifunctional $\mathrm{Ca}^{2+} /$ calmodulin-dependent protein kinase is conserved in the brain ryanodine receptor. J Biol Chem 267:4963-4967.

Wu LG, Betz WJ (1996) Nerve activity but not intracellular calcium determines the time course of endocytosis at the frog neuromuscular junction. Neuron 17:769-779.

Yamamoto N, Higashi S, Toyama K (1997) Stop and branch behaviors of geniculocortical axons: a time-lapse study in organotypic cocultures. J Neurosci 17:3653-3663.

Zhou FC (1990) Four patterns of laminin-immunoreactive structure in developing rat brain. Dev Brain Res 55:191-201. 